\section{The dissipation and release of proactive interference in a short-term memory task*}

\author{
RONALD H. HOPKINS†, RICHARD E. EDWARDS, and CHERI L, COOK \\ Washington State University, Pullman, Wash. 99163
}

In two experiments, each $S$ received four successive trials in a distractor-type short-term memory task. A rest period was interpolated between Trials 3 and 4 . Proactive interference (PI) developed across the first three trials and partially dissipated with rest. However, for all rest intervals tested (up to $5 \mathrm{~min}$ ), the recovery from PI was increased by a shift in taxonomic class. It was suggested that performance in the release-from-PI paradigm is mediated by a combination of long-term and short-term memory processes.

The encoding dimensions of verbal materials have recently received intensive investigation through the use of the release-from-PI (proactive interference) paradigm (Wickens, 1970 ). It has generally been assumed, either explicitly or implicitly, that the inferred encoding characteristics reflect short-term, as opposed to long-term, memory processes. However, Waugh \& Norman (1965) have suggested that even immediate retention is mediated by both long-term and short-term processes. By this argument, the encoding dimensions inferred from the release-from-PI experiments would not be exclusively attributable to short-term memory. The present experiments were designed to provide evidence concerning the locus of the PI that develops over successive trials of a short-term retention task.

The approach taken in the experiments reported here was to measure the time taken for PI to dissipate, once established. If the accrued PI were due to short-term processes, it should dissipate rapidly. We assumed that long-term processes would be implicated to the extent that the recovery from PI is not complete within a $2-\mathrm{min}$ rest period. The general procedure was very similar to that of Kincaid \& Wickens (1970), who found substantial recovery from PI after a $2-\mathrm{min}$ rest. The principal difference between the present procedure and that of Kincaid and Wickens was the inclusion of a series of control groups receiving, in addition to a rest interval,

\footnotetext{
*This research was supported by Basic $\mathrm{Research} G \mathrm{rant} A$ ward OEG-X-71-0032(508) from the United States Office of Education, by United States Public Health Service Grant MH 20232-01, and by Graduate School research funds. The second author was a United States National Aeronautics and Space Administration fellow during the conduct of this research. tRequests for reprints should be sent to Ronald H. Hopkins, Department of Ronald H. Hopkins, Department of
Psychology, Washington State University, Pullman, Wash. 99163.
}

a shift in taxonomic class of the to-be-remembered words. The experimental groups received only a rest interval. Since a taxonomic shift produces a marked release from PI (Loess, 1967), it was felt that the control groups would provide an index of the maximum amount of PI dissipation that might be expected in the experimental groups.

EXPERIMENT 1

Design and Materials

Each $S$ received four successive trials in a distractor-type short-term memory task. Half of the Ss received a shift in the taxonomic class of the to-be-remembered words between the third and fourth trials, and half did not. For each $S$, one of five rest intervals was interpolated between the third and fourth trials. Thus, there were 10 independent groups formed by the orthogonal combination of two levels of shift in taxonomic class (shift and no-shift) and five different lengths of rest interval $(0,15,30,60$, and $120 \mathrm{sec}$ ).

The stimulus words were selected from the animal-name and tree-name categories of the Battig \& Montague (1969) norms. The word triads formed from these materials were each homogeneous with respect to category and had approximately equal mean category frequency. Eight different lists of four triads each were formed, four for shift conditions and four for no-shift conditions. For the no-shift conditions, two lists contained four triads of animal names and two lists contained four triads of tree names. For the shift conditions, two lists contained three triads of animal names followed by one triad of tree names, and two lists contained three triads of tree names followed by one triad of animal names. The assignment of lists to rest-interval conditions was counterbalanced.

\section{Procedure}

The lists were presented to individual Ss via a Stowe memory drum. On each trial, the first advance of the drum exposed a triad of words for 5 sec. The second advance exposed a three-digit number for $15 \mathrm{sec}$; $S$ was instructed to say the number aloud and immediately begin counting backward by threes. The third advance of the drum exposed three asterisks for 5 sec as the signal for $S$ to recall the stimulus words aloud. The Ss were instructed to recall the words in their left-to-right order of presentation, and were encouraged to guess if uncertain. Immediately after the recall period, the word triad of the next trial was presented.

Following the third trial, the memory drum was stopped for 0,15 , 30,60 , or $120 \mathrm{sec}$. The $S$ was instructed to turn over a piece of paper and begin cancelling all of the $\mathrm{As}, \mathrm{Bs}$, and $\mathrm{Cs}$, according to instructions given at the beginning of the experiment. When told to stop, $\mathrm{S}$ placed his paper face down and looked back toward the window of the drum for the final trial.

\section{Subjects}

The Ss were 320 Washington State University undergraduates who participated in partial fulfillment of the requirements for an introductory psychology course. Thirty-two Ss were assigned to each condition according to a block-randomized order.

Results and Discussion

The intent was to score order of recall as well as item recall. However, an order error occurred on only $6 \%$ of the occasions where there was possibility for order error, so those data will not be reported. The mean numbers of words correctly recalled, regardless of order, are presented in Fig. 1 for each trial. On the first three trials there were no systematic differences in performance among the 10 groups, so those data have been pooled for clarity of presentation in the left-hand panel of Fig. 1. It may be seen that there was a marked decrement in performance from Trial 1 to Trial $3, F(2,638)=271.18$, $\mathrm{p}<.001$, indicating the development of considerable PI.

Trial 4 performance is shown in the right-hand panel of Fig. 1 for each of the 10 groups. There was some concern about ceiling effects in the shift groups. However, this does not seem to have been an important factor, as evidenced by the fact that the standard deviations were similar for all 10 groups, ranging from .84 to .98 for the shift groups and from .84 to 1.20 for the no-shift groups. Performance on Trial 4 did tend to improve as the rest interval was lengthened, $\mathrm{F}(4,310)=2.66, \mathrm{p}<.05$, indicating the dissipation of some PI. The shift conditions yielded better performance than the no-shift conditions, $F(1,310)=84.45$, $\mathrm{p}<.001$, and the interaction between 


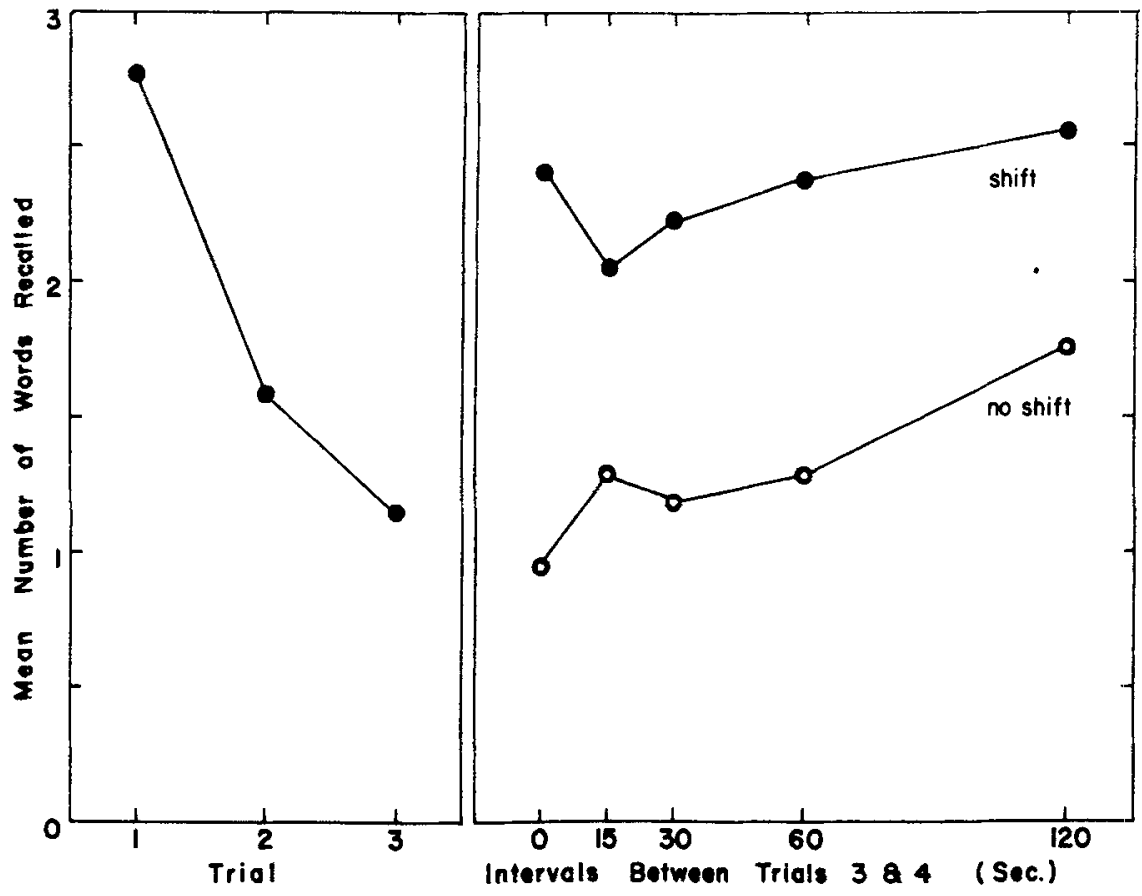

Fig. 1. Mean recall performance on Trials 1.4 of Experiment 1.

these two variables was not reliable, $\mathrm{F}(4,310)=1.20, \mathrm{p}>.20$. Thus, performance in the no-shift groups remained almost uniformly below that in the shift groups, indicating that substantial PI remained after a 2-min rest.

The dissipation of PI was also assessed by examining the improvement in performance with interpolated rest relative to the decrement in performance from Trial 1 to Trial 3 . The mean decrement in performance from Trial 1 to Trial 3 is 1.63. For the no-shift conditions, the mean improvement in performance is .81 from 0 to 120 sec of rest; obviously, this is only $50 \%$ of the Trial 1 to Trial 3 decrement. In contrast, Kincaid \& Wickens (1970) estiamted PI dissipation to be $74 \%$ complete after $120 \mathrm{sec}$. Thus, the present results seem to indicate a somewhat slower dissipation of PI than that obtained by Kincaid and Wickens. However, there is considerable difference in the amount of initial PI developed on Trials 1-3. Kincaid and Wickens used a 4-point scale and obtained a decrement from approximately 3.3 on Trial 1 to 2.3 on Trial 3, whereas the corresponding decrement in the present experiment was about 2.8 to 1.1 on a 3 -point scale. Also, Kincaid and Wickens obtained a mean improvement in performance of about .9 from 0 to 120 sec of rest. Thus, the percentage of PI recovery is different, but the absolute amount of PI dissipated is quite similar in the two experiments.
The amount of initial PI may be an important variable in the discrepancy in percentage of $P I$ recovery for the two experiments. However, the actual role of this variable is unknown and, furthermore, it is extremely difficult to manipulate the amount of initial PI without confounding by other variables.

Another difference between our experiment and that of Kincaid and Wickens, which might have influenced the rate of PI dissipation, is the difficulty of the rest-interval activity. Kincaid and Wickens used Stroop color naming, which is surely more difficult and distracting than our letter cancellation task. The importance of the difficulty of the rest activity was examined in Experiment 2 by comparing PI dissipation during a very easy task (sitting quietly) with that during a difficult activity (multiplication problems). Another: change made in Experiment 2 was to lengthen the rest interval to $5 \mathrm{~min}$, thus providing additional information regarding the rate of recovery from $\mathrm{PI}$.

\section{EXPERIMENT 2}

\section{Method}

The materials and procedure were identical to those of Experiment 1 with the following two exceptions. First, only one rest interval was used: $5 \mathrm{~min}$. Secondly, two different rest activities were used. Half of the Ss were given a sheet of 10 problems requiring the multiplication of a two-, three-, or four-digit number by another two-, three-, or four-digit number, and asked to complete as many as possible in the allotted time. The remaining Ss were given no rest activity and were simply asked to sit quietly. Thus, the design was a 2 by 2 factorial, with two levels of shift (shift and no shift) and two types of rest interval activity (none and multiplication). All $S$ s received four successive trials on the same lists as in Experiment 1, with a 5-min rest interval between Trials 3 and 4 . Thirty-two Ss, from the same pool at in Experiment 1, were assigned to each condition.

Results and Discussion

Overall mean recall scores on Trials 1,2 , and 3 were $2.84,1.66$, and 1.13 , respectively, almost identical to Experiment 1 . The means and standard deviations of Trial 4 recall scores are presented in Table 1 . It may be seen that Trial 4 performance in the shift groups was again superior to that in the no-shift groups, $F(1,124)=$ $5.85, p<.025$. The percentage of PI dissipation for the no-shift groups was approximately $69 \%$, estimated in the same manner as in Experiment 1. Thus, PI dissipation is only partially complete, even after $5 \mathrm{~min}$ of rest. Furthermore, the recovery from PI continues to be enhanced by a taxonomic shift for at least $5 \mathrm{~min}$. Finally, rest activity was not a reliable variable, either in main effect or in interaction with shift condition, both Fs $<1.0$. Thus, the differences between the present results and those of Kincaid and Wickens (1970) are probably not attributable to the difficulty of the rest interval activity. OVERALL DISCUSSION

Emphasis has been placed on the differences between the present results and those obtained by Kincaid \& Wickens (1970). One should, however, not lose sight of the similarities. In each case, a substantial proportion of the PI remains after a rest interval of 2 to $5 \mathrm{~min}$. Thus, some of the PI must be due to long-term, as opposed to short-term, memory processes. Equally important, however, is the finding that at least $50 \%$ of the PI does dissipate within $2 \mathrm{~min}$ and, therefore, can reasonably be attributed to short-term processes. The latter conclusion is quite different from the one reached by Craik \& Birtwistle (1971). Those investigators used a task requiring free recall of successive 15 -word lists and

Table 1

Mean Recall Scores on Trial 4 and Their Standard Deviations for the Conditions of Experiment 2

\begin{tabular}{lcccccr}
\hline & \multicolumn{2}{c}{ Shift } & & \multicolumn{2}{c}{ No Shift } \\
\cline { 2 - 3 } Rest Activity & $M$ & SD & & M & SD \\
\hline None & 2.66 & .83 & & 2.34 & .97 \\
Multiplication & 2.75 & .67 & & 2.31 & 1.00 \\
\hline
\end{tabular}


concluded that PI develops in the long-term store but not in the short-term store. On a theoretical level it may be assumed that the last few items of a free-recall list are retrieved from the short-term store; on an empirical level, however, the laws inferred from the free-recall task (used by Craik and Birtwistle) may be quite different from those inferred from the distractor task generally used in the release-from-PI paradigm.

On the basis of the present results and those obtained by Kincaid and Wickens, it appears that performance in the release-from-PI paradigm is mediated by a combination of long-term and short-term memory processes. It may, therefore, be necessary to develop alternative procedures for the study of encoding in short-term memory.

\section{REFERENCES}

BATTIG, W. F., \& MONTAGUE, W. E. Category norms for verbal items in 56 categories: A replication and extension of the Connecticut category norms. Journal of Experimental Psychology, 1969, 80(3, Pt. 2).

CRAIK, F. I. M., \& BIRTWISTLE, J.
Proactive inhibition in free recall. Journal of Experimental Psychology, 1971, 91, 120-123.

KINCAID, J. P., \& WICKENS, D. D Temporal gradient of release from proactive inhibition. Journal of Experimental Psychology, 1970, 86, 313-316.

LOESS, H. Short-term memory, word class, and sequence of items. Journal of Experimental Psychology, 1967, 74, 556-561.

WAUGH, N. C., \& NORMAN, D. A. Primary memory. Psychological Review, 1965, 72 , 89-104.

WICKENS, D. D. Encoding categories of words: An empirical approach to me aning. Psychological Review, 1970, 77. $1-15$. 